\title{
SOLVING SIMPLE QUATERNIONIC DIFFERENTIAL EQUATIONS
}

\author{
Stefano De Leo ${ }^{1}$ and Gisele C. Ducati ${ }^{2}$ \\ 1 Department of Applied Mathematics, State University of Campinas \\ PO Box 6065, SP 13083-970, Campinas, Brazil \\ deleo@ime.unicamp.br \\ 2 Department of Mathematics, University of Parana \\ PO Box 19081, PR 81531-970, Curitiba, Brazil \\ ducati@mat.ufpr.br \\ June, 2003
}

\begin{abstract}
The renewed interest in investigating quaternionic quantum mechanics, in particular tunneling effects [1], and the recent results on quaternionic differential operators [2] motivate the study of resolution methods for quaternionic differential equations. In this paper, by using the real matrix representation of left/right acting quaternionic operators, we prove existence and uniqueness for quaternionic initial value problems, discuss the reduction of order for quaternionic homogeneous differential equations and extend to the non-commutative case the method of variation of parameters. We also show that the standard Wronskian cannot uniquely be extended to the quaternionic case. Nevertheless, the absolute value of the complex Wronskian admits a non-commutative extension for quaternionic functions of one real variable. Linear dependence and independence of solutions of homogeneous (right) $\mathbb{H}$-linear differential equations is then related to this new functional. Our discussion is, for simplicity, presented for quaternionic second order differential equations. This involves no loss of generality. Definitions and results can be readily extended to the $n$-order case.
\end{abstract}

PACS. 02.10.Hh - 02.30.Tb MSC. 11R52-30G35-46S20

\section{INTRODUCTION}

Let $\mathbb{R}, \mathbb{C} \equiv \operatorname{span}\{1, i\}$, and $\mathbb{H} \equiv \operatorname{span}\{1, i, j, k\}$ be the real, complex , and quaternionic field [3],

$$
i^{2}=j^{2}=k^{2}=i j k=-1,
$$

and

$$
\mathcal{F}: \mathbb{R} \rightarrow \mathbb{R}
$$

be the set of real functions of real variable. Through the paper, quaternionic functions of real variable, $\Psi(x) \in \mathbb{H} \otimes \mathcal{F}$, will be denoted by Greek letter and constant quaternionic coefficients by Roman letter. To shorten notation the prime and double prime in the quaternionic functions shall respectively indicate the first and second derivative of quaternionic functions with respect to the real variable $x$,

$$
\Psi^{\prime}:=\frac{\mathrm{d} \Psi}{\mathrm{d} x} \quad \text { and } \quad \Psi^{\prime \prime}:=\frac{\mathrm{d}^{2} \Psi}{\mathrm{d} x^{2}} .
$$

Due to the non-commutative nature of quaternions, it is convenient to distinguish between the left and right action of the quaternionic imaginary units $i, j$, and $k$ by introducing the operators $L_{q}$ and $R_{p}$ whose action on quaternionic functions $\Psi$ is given by

$$
L_{q} \Psi=q \Psi \quad \text { and } \quad R_{p} \Psi=\Psi p,
$$


These (left/right acting) quaternionic operators satisfy

$$
L_{q} L_{p}=L_{q p}, \quad R_{q} R_{p}=R_{p q} \quad \text { and } \quad\left[L_{q}, R_{p}\right]=0
$$

and admit for

$$
q=q_{0}+i q_{1}+j q_{2}+k q_{3}, \quad p=p_{0}+i p_{1}+j p_{2}+k p_{3}, \quad \Psi=\Psi_{0}+i \Psi_{1}+j \Psi_{2}+k \Psi_{3},
$$

the following real matrix representation [4-6]

$$
L_{q} \leftrightarrow\left(\begin{array}{cccc}
q_{0} & -q_{1}-q_{2} & -q_{3} \\
q_{1} & q_{0} & -q_{3} & q_{2} \\
q_{2} & q_{3} & q_{0} & -q_{1} \\
q_{3}-q_{2} & q_{1} & q_{0}
\end{array}\right), \quad R_{p} \leftrightarrow\left(\begin{array}{cccc}
p_{0} & -p_{1} & -p_{2} & -p_{3} \\
p_{1} & p_{0} & p_{3} & -p_{2} \\
p_{2} & -p_{3} & p_{0} & p_{1} \\
p_{3} & p_{2} & -p_{1} & p_{0}
\end{array}\right), \quad \Psi \leftrightarrow\left[\begin{array}{c}
\Psi_{0} \\
\Psi_{1} \\
\Psi_{2} \\
\Psi_{3}
\end{array}\right] \in \mathbb{R}^{4} \otimes \mathcal{F}
$$

\section{EXISTENCE AND UNIQUENESS.}

In this section we discuss existence and uniqueness for the quaternionic initial value problem

$$
\Psi^{\prime \prime}=\alpha \Psi^{\prime}+\beta \Psi+\rho, \quad \Psi\left(x_{0}\right)=f, \quad \Psi^{\prime}\left(x_{0}\right)=g,
$$

with $\alpha(x), \beta(x), \rho(x) \in \mathbb{H} \otimes \mathcal{F}, x_{0} \in I:\left(x_{-}, x_{+}\right)$and $f, g \in \mathbb{H}$.

Theorem 1. Let $\alpha, \beta$ and $\rho$ in Eq.(4) be continuous functions of $x$ on an open interval I containing the point $x=x_{0}$. Then, the initial value problem (4) has a solution $\Psi$ on this interval and this solution is unique.

Proof. By using the real matrix representation (3), we can immediately rewrite the quaternionic initial value problem (4) in the following vector form

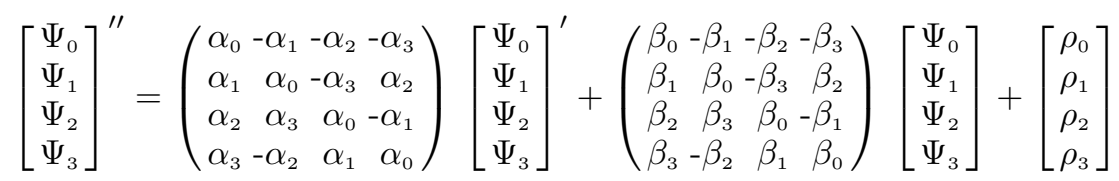

with

$$
\left[\begin{array}{l}
\Psi_{0}\left(x_{0}\right) \\
\Psi_{1}\left(x_{0}\right) \\
\Psi_{2}\left(x_{0}\right) \\
\Psi_{3}\left(x_{0}\right)
\end{array}\right]=\left[\begin{array}{l}
f_{0} \\
f_{1} \\
f_{2} \\
f_{3}
\end{array}\right] \text { and }\left[\begin{array}{l}
\Psi_{0}^{\prime}\left(x_{0}\right) \\
\Psi_{1}^{\prime}\left(x_{0}\right) \\
\Psi_{2}\left(x_{0}\right) \\
\Psi_{3}^{\prime}\left(x_{0}\right)
\end{array}\right]=\left[\begin{array}{l}
g_{0} \\
g_{1} \\
g_{2} \\
g_{3}
\end{array}\right]
$$

Eq.(5) represents a (nonhomogeneous) linear system with $\alpha_{m}, \beta_{m}, \rho_{m} \in \mathbb{R} \otimes \mathcal{F}$, where $m=0,1,2,3$. These functions are (see hypothesis of Theorem 1) continuous (real) functions of $x$ on an open interval $I$ containing the point $x=x_{0}$. Then, by a well-known theorem of analysis, see for example ref. [7], the linear system (5) has a solution

$$
\left[\begin{array}{l}
\Psi_{0} \\
\Psi_{1} \\
\Psi_{2} \\
\Psi_{3}
\end{array}\right] \in \mathbb{R}^{4} \otimes \mathcal{F}
$$

on this interval satisfying (6), and this solution is unique

\section{LINEAR INDEPENDENCE AND DEPENDENCE OF SOLUTIONS}

Let us now analyse the linear independence and dependence of the solutions of second order homogeneous differential equations

$$
\Psi^{\prime \prime}=\alpha \Psi^{\prime}+\beta \Psi
$$


where $\alpha$ and $\beta$ are (quaternionic) continuous functions of $x$ on an open interval I. Eq.(7) is linear over $\mathbb{H}$ from the right. Consequently, if $\varphi$ is a solution of Eq.(7) only the function obtained by right multiplication by constant quaternionic coefficients, $\varphi u$, still represent a solution of such an equation. The general solution of Eq.(7) is given in terms of a pair of linearly independent solutions $\varphi$ and $\xi$ by

$$
\Psi=\varphi u+\xi v,
$$

where $\varphi=\varphi_{0}+i \varphi_{1}+j \varphi_{2}+k \varphi_{3}, \xi=\xi_{0}+i \xi_{1}+j \xi_{2}+k \xi_{3} \in \mathbb{H} \otimes \mathcal{F}$ and $u, v \in \mathbb{H}$.

In the standard complex theory $\left(\varphi=\varphi_{0}+i \varphi_{1}\right.$ and $\left.\xi=\xi_{0}+i \xi_{1} \in \mathbb{C} \otimes \mathcal{F}\right)$ a useful criterion to establish linear independence and dependence of two solutions of homogeneous second order differential equation, uses the concept of Wronskian of these solutions defined by

$$
W=\varphi \xi^{\prime}-\varphi^{\prime} \xi, \quad W \in \mathbb{C} \otimes \mathcal{F} .
$$

This definition cannot be extended to quaternionic functions. Let us consider two linearly dependent solutions of Eq.(7),

$$
\xi=\varphi q, \quad \varphi, \xi \in \mathbb{H} \otimes \mathcal{F}, \quad q \in \mathbb{H} .
$$

By substituting $\xi=\varphi q$ and $\xi^{\prime}=\varphi^{\prime} q$ in the Wronskian (9), we find

$$
\varphi \xi^{\prime}-\varphi^{\prime} \xi=\varphi \varphi^{\prime} q-\varphi^{\prime} \varphi q \neq 0 .
$$

Observe that a quaternionic function and its first derivative do not, in general, commute. Thus, the definition (9), and all its possible factor combinations cannot be extended to the quaternionic case.

Let us now use the linear dependence condition (10) to investigate the possibility to define a quaternionic functional which extends (in a nontrivial way) the standard (complex) Wronskian to the noncommutative case. From Eq.(10) and its derivative, we get

$$
q=\varphi^{-1} \xi=\left(\varphi^{\prime}\right)^{-1} \xi^{\prime},
$$

where $\varphi^{-1} \equiv 1 / \varphi$ and $\left(\varphi^{\prime}\right)^{-1} \equiv 1 / \varphi^{\prime}$. Consequently, for linearly dependent quaternionic solutions , we have

$$
\xi^{\prime}-\varphi^{\prime} \varphi^{-1} \xi=0
$$

To recover, in the complex limit, the standard definition (9) we multiply $\xi^{\prime}-\varphi^{\prime} \varphi^{-1} \xi$ by $\varphi$. Due to the noncommutative nature of quaternions, we have to consider the following possibilities

$$
W_{L}=\varphi\left(\xi^{\prime}-\varphi^{\prime} \varphi^{-1} \xi\right) \quad \text { and } \quad W_{R}=\left(\xi^{\prime}-\varphi^{\prime} \varphi^{-1} \xi\right) \varphi .
$$

Obviously two other similar definitions can be obtained by $\varphi \leftrightarrow \xi$,

$$
\widetilde{W}_{L}=-\xi\left(\varphi^{\prime}-\xi^{\prime} \xi^{-1} \varphi\right)=-W_{L}[\varphi \leftrightarrow \xi] \quad \text { and } \quad \widetilde{W}_{R}=-\left(\varphi^{\prime}-\xi^{\prime} \xi^{-1} \varphi\right) \xi=-W_{R}[\varphi \leftrightarrow \xi] .
$$

The quaternionic functionals (12) and (13), which give in the complex limit the standard definition, extend a first important property of Wronskian. Two solutions of Eq.(7) are linearly dependent on I if $W_{L(R)}\left[\widetilde{W}_{L(R)}\right]$ is zero on I. To avoid ambiguity in defining the Wronskian, we shall introduce a (real) functional,

$$
|W|^{2}=\left|W_{L}\right|^{2}=\left|W_{R}\right|^{2}=\left|\widetilde{W}_{L}\right|^{2}=\left|\widetilde{W}_{R}\right|^{2},
$$

which extends the squared absolute value of the Wronskian. This unique functional is

$$
|W|^{2}=|\varphi|^{2}\left|\xi^{\prime}\right|^{2}+|\xi|^{2}\left|\varphi^{\prime}\right|^{2}-\varphi^{\prime} \varphi_{c} \xi \xi_{c}^{\prime}-\xi^{\prime} \xi_{c} \varphi \varphi_{c}^{\prime} \quad \in \mathbb{R} \otimes \mathcal{F},
$$

where $\varphi_{c}=\varphi_{0}-i \varphi_{1}-j \varphi_{2}-k \varphi_{3}$ and $\xi_{c}=\xi_{0}-i \xi_{1}-j \xi_{2}-k \xi_{3}$ are, respectively, the quaternionic conjugate functions of $\varphi$ and $\xi$.

Observe that Eq.(14) can also be obtained as an application of the Dieudonné theory of quaternionic determinants [8-12]. In fact,

$$
|W|^{2}=[\operatorname{Det}(M)]^{2}:=\operatorname{det}\left(M M^{+}\right),
$$


where

$$
M=\left(\begin{array}{cc}
\varphi & \xi \\
\varphi^{\prime} & \xi^{\prime}
\end{array}\right)
$$

Theorem 2. Let $\alpha$ and $\beta$ in Eq.(7) be continuous functions of $x$ on an open interval $I:(a, b)$. Then, two solutions $\varphi$ and $\xi$ of Eq.(7) on I are linearly dependent on I if and only if the absolute value of the Wronskian, $|W|$, is zero at some $x_{0}$ in I.

The proof will be divided into three steps:

(a) - If $\varphi$ and $\xi$ are linearly dependent on I then $|W|=0$;

(b) - If $|W|=0$ at some $x_{0}$ in I then $|W|=0$ on I.

(c) - If $|W|=0$ at some $x_{0}$ in I then $\varphi$ and $\xi$ are linearly dependent on I.

Proof (a). If $\varphi$ and $\xi$ are linearly dependent on I, then Eq.(10) holds on I. From Eq.(10), we get

$$
|W|^{2}=|\varphi|^{2}\left|\varphi^{\prime}\right|^{2}|q|^{2}+|\varphi|^{2}\left|\varphi^{\prime}\right|^{2}|q|^{2}-\varphi^{\prime} \varphi_{c} \varphi|q|^{2} \varphi_{c}^{\prime}-\varphi^{\prime}|q|^{2} \varphi_{c} \varphi \varphi_{c}^{\prime}=0,
$$

then $|W|=0$.

Proof (b). Let us consider Eq.(14). By calculating the first derivative of the left and right side term, we obtain

$$
\begin{aligned}
2|W||W|^{\prime}= & \varphi^{\prime} \varphi_{c} \xi^{\prime} \xi_{c}^{\prime}+\varphi \varphi_{c}^{\prime} \xi^{\prime} \xi_{c}^{\prime}+\varphi \varphi_{c} \Psi_{2}^{\prime \prime} \xi_{c}^{\prime}+\varphi \varphi_{c} \xi^{\prime} \xi_{c}^{\prime \prime}+ \\
& +\xi^{\prime} \xi_{c} \varphi^{\prime} \varphi_{c}^{\prime}+\xi \xi_{c}^{\prime} \varphi^{\prime} \varphi_{c}^{\prime}+\xi \xi_{c} \Psi_{1}^{\prime \prime} \varphi_{c}^{\prime}+\xi \xi_{c} \varphi^{\prime} \varphi_{c}^{\prime \prime}+ \\
& -\Psi_{1}^{\prime \prime} \varphi_{c} \xi \xi_{c}^{\prime}-\varphi^{\prime} \varphi_{c}^{\prime} \xi \xi_{c}^{\prime}-\varphi^{\prime} \varphi_{c} \xi^{\prime} \xi_{c}^{\prime}-\varphi^{\prime} \varphi_{c} \xi \xi_{c}^{\prime \prime}+ \\
& -\Psi_{2}^{\prime \prime} \xi_{c} \varphi \varphi_{c}^{\prime}-\xi^{\prime} \xi_{c}^{\prime} \varphi \varphi_{c}^{\prime}-\xi^{\prime} \xi_{c} \varphi^{\prime} \varphi_{c}^{\prime}-\xi^{\prime} \xi_{c} \varphi \varphi_{c}^{\prime \prime} \\
= & \left.\left|\varphi^{2}\left(\Psi_{2}^{\prime \prime} \xi_{c}^{\prime}+\xi^{\prime} \xi_{c}^{\prime \prime}\right)+\right| \xi\right|^{2}\left(\Psi_{1}^{\prime \prime} \varphi_{c}^{\prime}+\varphi^{\prime} \varphi_{c}^{\prime \prime}\right)+ \\
& -\Psi_{1}^{\prime \prime} \varphi_{c} \xi \xi_{c}^{\prime}-\varphi^{\prime} \varphi_{c} \xi \xi_{c}^{\prime \prime}-\Psi_{2}^{\prime \prime} \xi_{c} \varphi \varphi_{c}^{\prime}-\xi^{\prime} \xi_{c} \varphi \varphi_{c}^{\prime \prime} \\
= & |\varphi|^{2}\left(\alpha\left|\xi^{\prime}\right|^{2}+\beta \xi \xi_{c}^{\prime}+\text { h.c. }\right)+|\xi|^{2}\left(\alpha\left|\varphi^{\prime}\right|^{2}+\beta \varphi \varphi_{c}^{\prime}+\text { h.c. }\right)+ \\
& -\left[\left(\alpha \varphi^{\prime} \varphi_{c}+\beta|\varphi|^{2}\right) \xi \xi_{c}^{\prime}+\text { h.c. }\right]-\left[\left(\alpha \xi^{\prime} \xi_{c}+\beta|\xi|^{2}\right) \varphi \varphi_{c}^{\prime}+\text { h.c. }\right] \\
= & 2 \operatorname{Re}[\alpha]\left(|\varphi|^{2}\left|\xi^{\prime}\right|^{2}+|\xi|^{2}\left|\varphi^{\prime}\right|^{2}-\varphi^{\prime} \varphi_{c} \xi \xi_{c}^{\prime}-\xi^{\prime} \xi_{c} \varphi \varphi_{c}^{\prime}\right) \\
= & 2 \operatorname{Re}[\alpha]|W|^{2} .
\end{aligned}
$$

By a simple integration, we find

$$
|W(x)|=\exp \left[\int_{x_{0}}^{x} \operatorname{Re}[\alpha(y)] \mathrm{d} y\right]\left|W\left(x_{0}\right)\right| .
$$

This prove the statement (b).

Proof (c). From the statement (b), we have

$$
\left|W\left(x_{0}\right)\right|=0 \quad \Rightarrow \quad|W(x)|=0, \quad x \in \mathrm{I} .
$$

This implies that the quaternionic matrix

$$
\left(\begin{array}{ll}
\varphi & \xi \\
\varphi^{\prime} & \xi^{\prime}
\end{array}\right)
$$

is not invertible on I [12]. Hence the linear system

$$
\begin{aligned}
\varphi q_{1}+\xi q_{2} & =0, \\
\varphi^{\prime} q_{1}+\xi^{\prime} q_{2} & =0,
\end{aligned}
$$

in the unknowns $q_{1,2} \in \mathbb{H}$, has a solution $\left(q_{1}, q_{2}\right)$ where $q_{1}$ and $q_{2}$ are not both zero. Recalling that $\varphi$ and $\xi$ are linearly independent on an interval I if

$$
\varphi(x) q_{1}+\xi(x) q_{2}=0 \Rightarrow q_{1}=q_{2}=0,
$$


the fact that $q_{1}$ and $q_{2}$ are not both zero guarantees the linear dependence of $\varphi$ and $\xi$ on I

Example 1. Show that $\varphi=\exp [-i x]$ and $\xi=\exp [(i-j) x]$ form a basis of solutions of

$$
\Psi^{\prime \prime}+j \Psi^{\prime}+(1-k) \Psi=0,
$$

on any interval.

Solution. Substitution shows that they are solutions,

$$
\begin{aligned}
{[-1+j(-i)+1-k] \exp [-i x] } & =0, \\
{[-2+j(i-j)+1-k] \exp [(i-j) x] } & =0,
\end{aligned}
$$

and linear independence follows from Theorem 2, since

$$
|W|=\sqrt{|i-j|^{2}+|i|^{2}+i(j-i)-(i-j) i}=\sqrt{5} .
$$

\section{HOMOGENEOUS EQUATIONS: REDUCTION OF ORDER}

Let $\varphi$ be solution of Eq.(7) on some interval I. Looking for a solution in the form

$$
\xi=\varphi \tau
$$

and substituting $\xi$ and its derivatives

$$
\xi^{\prime}=\varphi^{\prime} \tau+\varphi \tau^{\prime} \quad \text { and } \quad \xi^{\prime \prime}=\varphi^{\prime \prime} \tau+2 \varphi^{\prime} \tau^{\prime}+\varphi \tau^{\prime \prime}
$$

into Eq.(7), we obtain

$$
\tau^{\prime \prime}=\left(\varphi^{-1} \alpha \varphi-2 \varphi^{-1} \varphi^{\prime}\right) \tau^{\prime} .
$$

It is important to observe that, for quaternionic functions, we cannot give a formal solution of the previous equation. Only in particular cases, Eq.(18) can be immediately integrated. For example, for homogeneous second order equations with constant coefficients,

$$
\alpha(x) \rightarrow a \in \mathbb{H} \text { and } \beta(x) \rightarrow b \in \mathbb{H},
$$

at least one solution is in the form of a quaternionic exponential, $\varphi=\exp [q x]$, and consequently Eq.(18) reduces to

$$
\varphi \tau^{\prime \prime}=(a-2 q) \varphi \tau^{\prime} .
$$

Let us introduce the quaternionic function

$$
\sigma=\varphi \tau^{\prime}
$$

Observing that

$$
\sigma^{\prime}=\varphi^{\prime} \tau^{\prime}+\varphi \tau^{\prime \prime}=q \varphi \tau^{\prime}+\varphi \tau^{\prime \prime}
$$

Eq.(19) can be rewritten as follows

$$
\sigma^{\prime}=(a-q) \sigma
$$

This equation can be immediately integrated, its solution reads

$$
\sigma=\exp [(a-q) x]
$$

Thus, the second solution of the homogeneous second order differential equation with constant coefficients is given by

$$
\xi=\exp [q x] \int \exp [-q x] \exp [(a-q) x] \mathrm{d} x .
$$

In the complex limit $(a, q \in \mathbb{C})$ we find the well-known results $\xi \propto \exp [(a-q) x]$ if $2 q \neq a$ and $\xi \propto x \exp [q x]$ if $2 q=a$. In the quaternionic case $(a, q \in \mathbb{C})$, the integral which appears in (21) 
must be treated with care. The solution of this integral will give interesting information about the second solution of quaternionic differential equations with constant coefficients when the associated characteristic quadratic equation has a unique solution. To solve the integral in Eq.(21), we start by observing that

$$
\left[e^{u x} e^{v x}\right]^{\prime}=u e^{u x} e^{v x}+e^{u x} e^{v x} v=\left(L_{u}+R_{v}\right) e^{u x} e^{v x} .
$$

If the operator $L_{u}+R_{v}$ is invertible the previous equality implies

$$
\int e^{u x} e^{v x} \mathrm{~d} x=\left(L_{u}+R_{v}\right)^{-1} e^{u x} e^{v x}
$$

This result guarantees that, if the operator $L_{-q}-R_{a-q}$ is invertible the second solution can be written in the form

$$
\begin{aligned}
\xi & =\exp [q x]\left(L_{-q}+R_{a-q}\right)^{-1} \exp [-q x] \exp [(a-q) x] \\
& =\exp [q x]\left(R_{a-q}-L_{q}\right)^{-1} \exp [-q x] \exp [(a-q) x]
\end{aligned}
$$

If the operator $L_{-q}+R_{a-q}$ is not invertible, we need to solve the integral which appears in (21) by using the polar decomposition of quaternions (see example 3) and a term linearly dependent on $x$ will appear. In the complex case $(a, q \in \mathbb{C})$, the operator $L_{-q}+R_{a-q}$ is not invertible if and only if $2 q=a$. In the quaternionic $(a, q \in \mathbb{H})$, the condition $2 q \neq a$ does not guarantee that the operator is invertible.

Example 2. Knowing that $\varphi=\exp [-i x]$ is solution of the homogeneous second order equation (17), find (by using the method of reduction of order) a second independent solution, $\xi$.

Solution. We have $q=-i$ and $a=-j$. To use Eq.(22) we have to prove that the operator

$$
L_{-q}+R_{a-q}=L_{i}+R_{i-j}
$$

is invertible. A simple algebraic calculation shows that

$$
\left(L_{i}-R_{i-j}\right)\left(L_{i}+R_{i-j}\right)=1 .
$$

Thus,

$$
\left(L_{-q}+R_{a-q}\right)^{-1}=L_{i}-R_{i-j} .
$$

We are now ready to calculate $\xi$ from Eq.(22),

$$
\xi=\exp [-i x]\left(L_{i}-R_{i-j}\right) \exp [i x] \exp [(i-j) x]=\left(L_{i}-R_{i-j}\right) \exp [(i-j) x]=\exp [(i-j) x] j .
$$

Due to the $\mathbb{H}$ linearity (from the right) of Eq.(17) the right factor $j$ can be ignored recovering the solution of example 1 .

Example 3. Inspection shows that

$$
\Psi^{\prime \prime}+i \Psi^{\prime}+\frac{k}{2}=0
$$

has $\varphi=\exp \left[-\frac{i+j}{2} x\right]$ as a first solution. Find the second linear independent solution.

Solution. We have $q=-\frac{i+j}{2}$ and $a=-i$. In this case, the operator

$$
L_{-q}+R_{a-q}=L_{\frac{i+j}{2}}+R_{\frac{j-i}{2}}
$$

is not invertible. This is easily seen by using, for example, the real matrix representation (3). Thus, the integral in Eq.(21) cannot be expressed in terms of an exponential product. Let us explicitly calculate $\xi$ from Eq.(21). We find

$$
\begin{aligned}
\xi & =\exp \left[-\frac{i+j}{2} x\right] \int \exp \left[\frac{i+j}{2} x\right] \exp \left[\frac{j-i}{2} x\right] \mathrm{d} x \\
& =\exp \left[-\frac{i+j}{2} x\right] \int\left(\cos \frac{x}{\sqrt{2}}+\frac{i+j}{\sqrt{2}} \sin \frac{x}{\sqrt{2}}\right)\left(\cos \frac{x}{\sqrt{2}}+\frac{j-i}{\sqrt{2}} \sin \frac{x}{\sqrt{2}}\right) \mathrm{d} x \\
& =\exp \left[-\frac{i+j}{2} x\right] \int\{1-k \exp [-(i+j) x]\} \frac{1+k}{2} \mathrm{~d} x .
\end{aligned}
$$


Due to the $\mathbb{H}$ linearity (from the right) of Eq.(23) the right factor $\frac{1+k}{2}$ can be removed. After integration, we find

$$
\xi=\exp \left[-\frac{i+j}{2} x\right]\left\{x-k \frac{i+j}{2} \exp [-(i+j) x]\right\}=\left(x+\frac{i-j}{2}\right) \exp \left[-\frac{i+j}{2} x\right] .
$$

Observe that the quaternionic factor $\frac{i-j}{2}$ appears on the left of the quaternionic exponential and consequently cannot be removed. It is a fundamental part of the solution. Inspection shows that

$$
\xi=x \exp \left[-\frac{i+j}{2} x\right]
$$

is not solution of Eq.(23).

\section{NONHOMOGENEOUS EQUATIONS: VARIATION OF PARAMETERS}

A general solution of the nonhomogeneous equation (4) is a solution of the form

$$
\Psi=\Psi_{h}+\Psi_{p}
$$

where

$$
\Psi_{h}=\varphi q_{1}+\xi q_{2}
$$

is a general solution of the homogeneous equation (7) and $\Psi_{p}$ is any particular solution of (4) containing no arbitrary constants. In this section we discuss the so-called method of variation of parameters to find a particular solution for quaternionic nonhomogeneous differential equations.

A method to solve a homogeneous second order quaternionic differential equations with constant coefficients has been recently developed [2]. Quaternionic differential equations with non constant coefficients are under investigation. We suppose to know two independent solutions of the homogeneous equation associated with Eq.(7). We wish to investigate if the method of variation of parameters still works in the quaternionic case.

The method of variation of parameters involves replacing the constant $q_{1}$ and $q_{2}$ by quaternionic functions $\nu_{1}(x)$ and $\nu_{2}(x)$ to be determined so that the resulting function

$$
\Psi_{p}=\varphi \nu_{1}+\xi \nu_{2}
$$

is a particular solution of Eq.(4). By differentiating $\Psi_{p}$ we obtain

$$
\Psi_{p}^{\prime}=\varphi^{\prime} \nu_{1}+\xi^{\prime} \nu_{2}+\varphi \nu_{1}^{\prime}+\xi \nu_{2}^{\prime}
$$

The requirement that $\Psi_{p}$ satisfies Eq. (4) imposes only one condition on $\nu_{1}$ and $\nu_{2}$. Hence, we can impose a second arbitrary condition, that is

$$
\varphi \nu_{1}^{\prime}+\xi \nu_{2}^{\prime}=0
$$

This reduces $\Psi_{p}^{\prime}$ to the form

$$
\Psi_{p}^{\prime}=\varphi^{\prime} \nu_{1}+\xi^{\prime} \nu_{2}
$$

By differentiating this function we have

$$
\Psi_{p}^{\prime \prime}=\varphi^{\prime \prime} \nu_{1}+\varphi^{\prime} \nu_{1}^{\prime}+\xi^{\prime \prime} \nu_{2}+\xi^{\prime} \nu_{2}^{\prime}
$$

Substituting $\Psi_{p}, \Psi_{p}^{\prime}$, and $\Psi_{p}^{\prime \prime}$ in Eq.(4) we readily obtain

$$
\varphi^{\prime} \nu_{1}^{\prime}+\xi^{\prime} \nu_{2}^{\prime}=\rho
$$

Collecting Eq.(25) and Eq.(26), we can construct the following matrix system

$$
\left(\begin{array}{cc}
\varphi & \xi \\
\varphi^{\prime} & \xi^{\prime}
\end{array}\right)\left[\begin{array}{l}
\nu_{1}^{\prime} \\
\nu_{2}^{\prime}
\end{array}\right]=\left[\begin{array}{l}
0 \\
\rho
\end{array}\right],
$$


from which $(|W| \neq 0)$ we obtain

$$
\begin{aligned}
{\left[\begin{array}{l}
\nu_{1}^{\prime} \\
\nu_{2}^{\prime}
\end{array}\right] } & =\left(\begin{array}{cc}
\varphi & \xi \\
\varphi^{\prime} & \xi^{\prime}
\end{array}\right)^{-1}\left[\begin{array}{l}
0 \\
\rho
\end{array}\right] \\
& =\left(\begin{array}{l}
{\left[\varphi-\xi \xi^{\prime-1} \varphi^{\prime}\right]^{-1}\left[\varphi^{\prime}-\xi^{\prime} \xi^{-1} \varphi\right]^{-1}} \\
{\left[\xi-\varphi \varphi^{\prime-1} \xi^{\prime}\right]^{-1}\left[\xi^{\prime}-\varphi^{\prime} \varphi^{-1} \xi\right]^{-1}}
\end{array}\right)\left[\begin{array}{l}
0 \\
\rho
\end{array}\right]
\end{aligned}
$$

Then,

$$
\nu_{1}^{\prime}=\left[\varphi^{\prime}-\xi^{\prime} \xi^{-1} \varphi\right]^{-1} \rho \quad \text { and } \quad \nu_{2}^{\prime}=\left[\xi^{\prime}-\varphi^{\prime} \varphi^{-1} \xi\right]^{-1} \rho .
$$

To find $\nu_{1}(x)$ and $\nu_{2}(x)$ we have to integrate the previous equations.

Example 4. Find a general solution of the nonhomogeneous quaternionic differential equation

$$
\Psi^{\prime \prime}+j \Psi^{\prime}+(1-k) \Psi=i x
$$

Solution. The solution of the associated homogeneous equation (see example 1) is

$$
\Psi_{h}=\exp [-i x] q_{1}+\exp [-(i+j) x] q_{2} .
$$

The particular solution is

$$
\Psi_{p}=\exp [-i x] \nu_{1}+\exp [-(i+j) x] \nu_{2} .
$$

Consequently, from Eqs.(29) we find

$$
\nu_{1}^{\prime}=\exp [i x] x k \quad \text { and } \quad \nu_{2}^{\prime}=-\exp [(i+j) x] x k
$$

which after integration give

$$
\nu_{1}(x)=(1-i x) \exp [i x] k \quad \text { and } \quad \nu_{2}(x)=-\frac{1}{2}[1-(i+j) x] \exp [(i+j) x] k .
$$

Finally

$$
\Psi_{p}=\frac{1}{2}[(i+j) x+k]
$$

A general solution of Eq.(23) is

$$
\Psi=\exp [-i x] q_{1}+\exp [-(i+j) x] q_{2}+\frac{1}{2}[(i+j) x+k] .
$$

\section{VI.CONCLUSIONS AND OUTLOOKS}

The recent results on violations of quantum mechanics by quaternionic potentials [1] and the possibility to get a better understanding of $\mathrm{CP}$-violation phenomena within a quaternionic formulation of physical theories $[1,13]$ stimulated the study of quaternionic differential operators [2]. In this paper, we have proved existence and uniqueness for quaternionic initial value problems and solved simple quaternionic differential equations by discussing the reduction of order for quaternionic homogeneous equations and by extending to the non-commutative case the method of variation of parameters and the definition of absolute value of the Wronskian functional.

In view of a more complete discussion of quantum dynamical systems using quaternionic wave packets, our next research (mathematical) interest will be the study of quaternionic integral transforms. The quaternionic formulation of Fourier transforms could find an immediate and interesting application in the study of delay time modifications of wave packets scattered by a quaternionic potential step.

\section{Acknowledgements}

The authors thank the referee for comments, references, and suggestions, and for drawing their attention to an alternative way to obtain the squared absolute value of the Wronskian (14) by Diuedonné's theory of determinants [see Eq.(15)]. 


\section{References}

1. S. De Leo, G. C. Ducati and C. C. Nishi, Quaternionic potentials in non relativistic quantum mechanics, J. Phys. A 35, 5411-5426 (2002).

2. S. De Leo and G. C. Ducati, Quaternionic differential operators, J. Math. Phys. 42, 2236-2265 (2001).

3. W. R. Hamilton, Elements of Quaternions, vol I, $3^{\text {rd }}$ Ed, Chelsea Publishing Co., New York (1969).

4. S. De Leo and G. C. Ducati, Quaternionic groups in physics, Int. J. Theor. Phys. 38, 2197-2220 (1999).

5. S. De Leo and G. Scolarici, Right eigenvalue equation in quaternionic quantum mechanics, J. Phys. A 33, 2971-2995 (2000).

6. S. De Leo, G. Scolarici and L. Solombrino, Quaternionic eigenvalue problem, J. Math. Phys. 43, 5815-5829 (2002).

7. E. Kreyszig, Advanced Engineering Mathematics (John Wiley \& Sons, New York, 1993), pag. 165.

8. E. Study, Zur Theorie der linear Gleichugen, Acta Math. 42, 1-62 (1920).

9. J. Brenner, Applications of the Diuedonné determinant, Linear Algebra Appl. 1, 511-536 (1968).

10. F. J. Dyson, Quaternion determinants, Helv. Phys. Acta 45, 289-302 (1972).

11. H. Aslaksen, Quaternionic determinants, Math. Intelligencer 18, 57-65 (1996).

12. N. Cohen and S. De Leo, The quaternionic determinant, Elec. J. Lin. Alg. 7, 100-111 (2000).

13. S. Adler, Quaternonic Quantum Mechanics and Quantum Fields (Oxford University Press, New York, 1995). 